\title{
Contemporary Review on Technologies and Methods for Converting Unstructured Data to Structured Data
}

\author{
Sreenivasulu Bolla ${ }^{1}$, R. Anandan ${ }^{2 *}$ \\ ${ }^{I}$ Department of Computer Science and Engineering, Vels Institute of Science, Technology and Advanced Studies (VISTAS), Chennai, \\ India.E-mail:sreenivasb8@gmail.com \\ ${ }^{2}$ Department of Computer Science \& Engineering, Vels Institute of Science, Technology and Advanced Studies (VISTAS), Chennai, India. \\ *Corresponding author E-mail:anandan.se@velsuniv.ac.in
}

\begin{abstract}
It is now evident that Big Data has pinched immense contemplation from scientists in data sciences, strategy and chiefs in governments and activities. As the speed of data progress surpasses Moore's Law toward the start of this new century, exorbitant data is making awesome inconveniences to individuals. Be that as it may, there are so much potential and exceedingly helpful esteems covered up in the tremendous volume of data. Be that as it may, huge data significantly contains three classes of data those are organized data, semi organized data, unstructured data. In this paper we are examining the diverse qualities and advancements of Big Data and predominantly concentrating on change of organized data to unstructured data and distinctive innovations required to changing over the data.
\end{abstract}

Keywords: Structured data, un-structured data, data extraction, data ordering, hadoop, mapreduce.

\section{Introduction}

A right importance of "Big data" is difficult to nail down in light of the way that endeavors, venders, specialists, and business specialists use it in a sudden way. In perspective of that, generally speaking, tremendous data is: immense datasets the characterization of preparing procedures and progressions [1] that are used to manage generous datasets.

In this one of a kind situation, "big dataset" suggests a dataset[2] excessively huge, making it impossible to sensibly process or store with ordinary tooling or on a singular PC. This infers the fundamental size of gigantic datasets is consistently moving and may vacillate basically from relationship to affiliation[5].

\section{Characteristics of Big Data}

\section{Volume}

The sheer size of the data dealt with portrays gigantic data systems. These datasets can be solicitations of size greater than standard datasets[7], which asks for more thought at each period of the taking care of and limit life cycle.

As often as possible, in light of the fact that the work necessities outperform the capacities of a singular PC, this transforms into a trial of pooling[4], assigning, and arranging resources from social events of PCs. Gathering organization and estimations fit for breaking errands into smaller pieces end up being logically crucial.

\section{Velocity}

Data being occasionally spilling keen on the classification on or after various sources and is regularly usual that would be arranged logically to get bits of learning and invigorate the present appreciation of the structure.

This accentuation on close minute info has driven various gigantic data experts from a group arranged approach and more like a constant spouting system[9]. Data is persistently being incorporated, plied, taken care of, and analyzed to remain mindful of the merging of new data and to surface noteworthy data early when it is by and large appropriate. These considerations require lively structures with exceedingly available parts to make arrangements for frustrations[11] along the data pipeline.

\section{Variety}

Another path by which colossal data moves on an exceptionally essential level from other data frameworks is the speed that data experiences the structure[8]. Data is every so often spilling into the framework from different sources and is consistently expected that would be orchestrated coherently to get bits of learning and empower the present valuation for the structure.

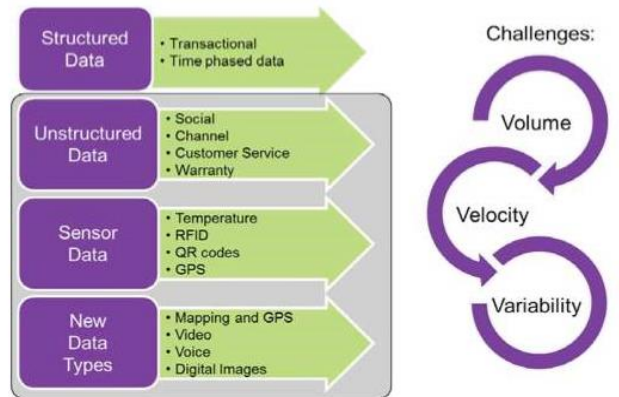

Fig. 1: Big data types and challenges

This emphasis on close moment data has driven different huge data specialists from a gathering masterminded approach and more like a consistent gushing framework[13]. Data is diligently 
being fused, employed, dealt with, and investigated to stay aware of the converging of new data and to surface critical data early when it is all around proper. These contemplations require energetic structures with exceedingly accessible parts to make plans for disappointments along the data pipeline.

\section{Big Data Life Cycle}

The general classifications of exercises required with Big Data preparing are:

- Ingesting data into the framework

- Continuing the data away

- Registering and Analyzing data

- Picturing the outcomes

Setting up a registering bunch is frequently the establishment for innovation utilized as a part of each of the life cycle stages.

In light of the characteristics of Big Data, singular PCs are regularly lacking for taking care of the data at generally arranges. To better address the high stockpiling and computational needs[10] of huge data, PC bunches are a superior fit.

The collected registering group regularly goes about as an establishment which other programming interfaces with to process the data. The machines associated with the registering bunch are additionally regularly included with the administration of a circulated stockpiling framework[13], which we will discuss when we talk about data industriousness.

\section{Persisting the Data in Storage}

The ingestion forms commonly hand the data off to the segments that oversee stockpiling, so it can be dependably persevered to plate. While this appears like it would be a basic operation, the volume of approaching data[15], the prerequisites for accessibility, and the appropriated figuring layer make more perplexing stockpiling frameworks vital.

This ordinarily implies utilizing a conveyed document framework for crude data stockpiling. Arrangements like Apache Hadoop's HDFS file system enable huge amounts of data to be composed over different hubs in the bunch. This guarantees the data can be gotten to by register assets, can be stacked into the group's RAM for in-memory operations[19], and can smoothly deal with segment disappointments. Other disseminated file systems can be utilized as a part of rest of HDFS counting Ceph and GlusterFS.

\section{Data Calculating and Analyzing}

Cluster preparing is one strategy for processing over an extensive dataset. The procedure includes separating work into littler pieces, booking each piece on an individual machine[12], reshuffling the data in view of the transitional outcomes, and afterward computing and collecting the last outcome. These means are regularly alluded to independently as part, mapping, rearranging [9][17], lessening, and gathering, or all things considered as a conveyed delineate calculation. This is the methodology utilized by Apache Hadoop's MapReduce. Clump handling is most valuable when managing huge datasets that require a lot of calculation[2].

Apache Storm, Apache Flink, and Apache Spark give distinctive methods for accomplishing ongoing or close constant preparing. There are exchange offs with each of these advances, which can influence which approach is best for any individual issue. When all is said in done, ongoing handling is most appropriate for breaking down littler lumps of data that are changing or being added to the framework quickly.

The above illustrations speak to computational structures. Be that as it may, there are numerous different methods for registering over or examining data inside a major data framework. These devices much of the time connect to the above systems and give extra interfaces to communicating with the hidden layers. For example, Apache Hive gives an data distribution center interface to Hadoop, Apache Pig gives an abnormal state questioning interface, while SQL-like connections with data can be accomplished with ventures like Apache Drill, Apache Impala, Apache Spark SQL, and Presto. For machine learning, ventures like Apache SystemML, Apache Mahout, and Apache Spark's MLlib can be helpful.

\section{Related Work}

Representation innovation normally utilized for intuitive data science work is an data "note pad". These ventures consider intelligent investigation and perception of the data in a configuration helpful for sharing, exhibiting, or working together. Mainstream cases of this sort of perception interface are Jupyter Notebook and Apache Zeppelin[6].

In the beginning of Google seek, engineers required an approach to store and recover the information in a proficient way that would scale to substantial sizes. In 2003, the exceptionally respected group centered Doug Cutting made an open source variant of the structure called Hadoop[9]. Hadoop is made by Doug Cutting and Mike Cafarella in 2005, created to help dissemination for the Nutch web search tool venture. Hadoop requires Java Runtime Environment (JRE) 1.6 or higher forms. The standard startup and shutdown content obliges secure shell to be set up between hubs and bunch. It comprises of OS level Abstractions[3], for example, MapReduce Engine and Hadoop Distributed File Framework (HDFS). HDFS is a record System written in Java Hadoop Framework. The Evolution of Hadoop has a fervent improvement in the field of huge information. Hadoop underpins the structure of Big Data as it is a parallel programming stage.

\section{Dictionary of Big Data}

While we've endeavored to characterize ideas as we've utilized them all through the guide, here and there it's useful to have particular phrasing accessible in a solitary place:

Big Data: Big data is a term for data sets that can't sensibly be dealt with by customary PCs or instruments because of their volume, speed, and assortment[7]. This term is likewise ordinarily connected to advances and procedures to work with this sort of data.

Bunch preparing: Batch handling is a registering technique that includes preparing data in substantial sets. This is ordinarily perfect for non-time touchy work that works on extensive arrangements of data[13]. The procedure is begun and at a later time, the outcomes are returned by the framework.

Data distribution centre: Data stockrooms are substantial, requested stores of data that can be utilized for examination and detailing. As opposed to an data lake, an data distribution center is made out of data that has been cleaned, coordinated with different sources[16], and is by and large very much requested. Data distribution centres are regularly talked about in connection to Big Data, however commonly are segments of more traditional frameworks.

Hadoop: Hadoop is an open source achievement in Big Data. It comprises of a disseminated file system called HDFS, with a group administration and asset which is on high level called YARN. Cluster preparing capacities are given by the Map Reduce calculation motor.

Machine learning: Machine learning is the examination and routine with regards to planning frameworks that can learn, modify, and enhance in view of the data sustained to them[5]. This normally includes usage of prescient and factual calculations that can constantly focus in on "redress" conduct and bits of knowledge as more data courses through the framework.

NoSQL: NoSQL is an expansive term alluding to databases outlined outside of the customary social model. NoSQL databases have distinctive exchange offs contrasted with social 
databases[11], yet are regularly appropriate for huge data frameworks because of their adaptability and continuous conveyed first engineering.

Stream preparing: Stream handling is the act of registering over individual data things as they travel through a framework. This takes into consideration continuous investigation of the data being sustained to the framework and is helpful for time-delicate operations utilizing high speed measurements.

\section{Conversion of Unstructured Data to Structured Data}

Nowadays, Big Data is depicted with 3 words volume, speed and assortment. The thought or idea to manufacture the creating forms so as to deal with the expanding 'volumes' and 'speed' of learning almost looks attainable. Be that as it may, from a strategy perfection reason we are particularly inquisitive[15] about the 'assortment', as this identifies with two data class; organized data learning and unstructured data. The web data extraction administrations are utilized to remove both of this data writes to be connected for business and innovation purposes.

Unstructured data is a nonexclusive term to portray learning that does not sit in knowledgebase's and might be a blend of printed and non-literary data. It is hard to change over unstructured data to organized[18] data as it for the most part lives in media like messages, reports, introductions, spreadsheets, pictures, video or sound records.

As the volumes of this kind of data have expanded through the work of good innovation the need to dissect this data and its mindfulness has additionally developed. This unstructured data document is handled and changed over into organized data as the yield by utilizing unstructured data to organized data transformation instruments. Computerized unstructured data mining programming will without a doubt help in such situations.

\section{Transforming Unstructured Data to Structured Data}

The most effective method to change over unstructured data to organized data in Hadoop with an illustration. A tremendous aspect regarding Hadoop is that it gives a steady, simple on the pocket and nearly a less complex system for social affair, restricting and putting away numerous data streams that was a few years back not achievable.

Taking a case, think about unstructured data in Hadoop just like a raw petroleum. Despite the fact that it is a standout amongst the most significant crude materials, however before you can concentrate or get required fuel from rough we require to put it over a sifting[7] or more exact a refining technique in a refinery to evacuate its polluting influence, and concentrate the profitable hydrocarbons which can be ordered as organized data[9].

Organized data is moderately uncomplicated and simple to use, Utilizing organized data is simple with its methodological improvements and as they live in databases inside the classification of lines and sections. It's grouped into relations or classes construct for the most part upon shared attributes[15]. The data is generally apportioned properties (data depictions) related with the classifications inside each bunch to help with requesting and legitimately gathering[7]. At last it is regularly portrayed by predefined groups (string or esteem) with predefined lengths of characters.

This makes organized data a conventional place to start for anybody aching for strong learning to shape data upon that to make noteworthy bits of knowledge[10]. Organized data are frequently questioned and dissected to type, gathering, channel, consider and add up to so to answer business inquiries or live technique ability. It is utilized as a part of item data knowledge and in addition value checking programming arrangements[3].
With the record for the legitimacy of the data it modifies relatively with the procedure to check and watch the data. Organized data shapes an out-sized piece of the data used by a few in technique improvements, however this pattern is rapidly dynamical in light of the fact that the strength of unstructured data will increment. Unstructured data extraction include complexities while preparing the data at first as unstructured data lives on organization systems, inside joint effort apparatuses and inside the cloud these are regularly extremely troublesome[8] to question. To look the data, forms should be set up to help tag and sort it. This progression is fundamental to allow for etymology looking against catchphrases or settings.

Unstructured learning is being utilized as a part of an exceedingly gigantic approach for web-based social networking organizations expecting to see their business sectors and clients in extra profundity[15]. This presents indistinguishable chances to a few of our organizations to help see not exclusively its clients higher, however operations inside.

A current IDC report anticipated the measure of advanced substance in 2012 can increment from 2011 figures by forty eighth percent to more than 2.7 zeta bytes (ZB) kept on partner 7.9 zeta bytes (ZB) by 2015 . More than $90 \%$ of this data is measurable to be unstructured data that features the need to create strong techniques to know and investigate the installed data.

Difficulties with Business Processes in connection to unstructured data extraction

The test for organizations is to create procedures to utilize structure to the unstructured idea of the data for example vital the measure of fulfillment of shoppers by breaking down messages and online networking could include dealing with words or expressions[13]. Words and expressions could likewise be grouped into positive, negative or impartial arrangements.

At this stage the unstructured data is renovated to organized learning by utilizing unstructured data mining programming wherever the groups of words discovered construct for the most part upon their arrangement are relegated an esteem[3]. A positive word could parallel one, a negative - 1 and an unbiased zero. This unstructured data will right now be kept and broke down as you'd with organized learning. Or maybe more work is required amid this space to dissect the unstructured data and a lot of the vast sellers are working on arrangements.

I trust the organizations that may get the first of their unstructured data sources are the individuals who see ways and unstructured data mining programming [8] devices to redesign the unstructured to organized data.

\section{Unstructured Data to Structured Data Tools}

Unstructured data extraction instruments accessible:

\section{Upstream Commerce}

Upstream Commerce offers retailers answers for help business edges through focused data bits of knowledge, valuing insight and item grouping. It has helped associations crosswise over retailing classifications to upgrade valuing, advancement and marketing administration[2]. It enables business clients to construct insight by utilizing examples and associations with both organized and unstructured data extractions.

\section{Data Crops}

Data Crops is an adaptable programming stage that brilliantly separates data from various sites and confused online data sources by utilizing a vigorous self-upgraded innovation. It extricates data, change and load it, guaranteeing the conveyance of right data at amend time and in a required and right arrangement[7]. 
Data Crops offers web data extraction arrangements and explanatory apparatuses to remove data utilizing both organized and unstructured data sources[14]. It removes unstructured data and change over it into business experiences to enable retail, to movement, inns, flight, tire, explore, back, data administration and online market insight organizations. Alongside web data extraction programming arrangements[9], it additionally offers value knowledge devices, item insight, online market knowledge, web-based social networking knowledge, mark knowledge and channel knowledge arrangements.

\section{Parse Hub}

Parse Hub is a visual data extraction apparatus for getting web data. It oversees intelligent timetables, maps, look, settled remarks, dropdowns, shapes, unending looking over, verification, discussions[16], Ajax, JavaScript, and considerably more effortlessly. You can make APIs from various locales utilizing this apparatus. Parse Hub gives different plans to web data extraction.

\section{Talend Data Fabric}

Talend Data Fabric is an data joining stage that gives customers a chance to work between spilling, clump, and continuous data. It keeps running on-premises, in Cloud and with Big Data[12][17]. Talend offers its clients an extraordinary plan interface for all data mixes and the ace data administration prerequisites. [22]

\section{ABBYY Flexi Capture}

ABBYY Flexi Capture is an data catching, extraction and record preparing programming apparatus for unstructured data examination. It is all around intended to transmute surges of records of organized, unstructured and multifaceted nature into business data[7]. It offers programmed data extraction from various solicitations and fare it to online sources.

It offers modified archive grouping, with an adaptable and adjustable engineering, helping organizations of any size to modernize their business forms, upsurge proficiency and reduction costs. It removes data from reports, content substantial papers, organized structures and the overviews.[23]

\section{Conclusion}

As we have entered a time of Big Data which is the following outskirts for development, rivalry and profitability, another rush of logical upheaval is going to start. Luckily, we will witness the coming innovative jumping. In this review paper, we give a concise outline on Big Data issues, including Big Data openings and difficulties, current procedures and innovations. We additionally propose a few potential strategies to changing over unstructured data to organized data.

\section{References}

[1] Gantz J \& Reinsel D, "Extracting value from chaos", IDC Iview, (2011)

[2] Boudreau MC \& Robey D, "Enacting integrated data technology: a human agency perspective", Organ. Sci., Vol.16, (2005), pp.3-15.

[3] Wand Y \& Weber R, "On the deep structure of data systems", Inf. Syst. J., Vol.5, (1995), pp.203-223.

[4] Manju, K., Sabeenian, R. S., \& Surendar, A. (2017). A review on optic disc and cup segmentation. Biomedical and Pharmacology Journal, 10(1), 373-379

[5] DeSanctis G \& Poole MS, "Capturing the complexity in advanced technology use: adaptive structuration theory", Organ. Sci., Vol.5, (1994), pp.121-147.

[6] Burton-Jones A \& Grange C, "From use to effective use: a representation theory perspective", Inf. Syst. Res., Vol.24, (2012), pp.632-658
[7] Berg M \& Goorman E, "The contextual nature of medical data", Int. J. Med. Inform., Vol.56, (1999), pp.51-60.

[8] Berg M, "Implementing data systems in health care organizations: myths and challenges", Int. J. Med. Inform., Vol.64, (2001), pp.143-156.

[9] Eveleigh A, Jennett C, Blandford A, Brohan P \& Cox AL, "Designing for dabblers and deterring drop-outs in citizen science", Proceedings of the 32nd Annual ACM Conference on Human Factors in Computing Systems, (2014), pp.2985-2994.

[10] Burton-Jones A \& Volkoff O, "How can we develop contextualized theories of effective use? A demonstration in the context of community-care electronic health records", Inf. Syst. Res., (2017).

[11] Lukyanenko R \& Parsons J, "Data quality research challenge: adapting dataquality principles to user-generated content", J. Data Inf. Qual. (JDIQ), Vol.6, No.3, (2015).

[12] Tremblay MC, Berndt DJ, Luther SL, Foulis PR \& French DD, "Identifying fall-related injuries: text mining the electronic medical record", Inf. Technol. Manage., Vol.10, (2009), pp.253-265.

[13] Sørlie T, Perou CM, Tibshirani R, Aas T, Geisler S, Johnsen H, Hastie T, Eisen MB, van de Rijn M \& Jeffrey SS, "Gene expression patterns of breast carcinomas distinguish tumor subclasses with clinical implications", Proc. Natl. Acad. Sci., Vol.98, (2001), pp.10869-10874.

[14] Larsen K \& Bong CH, "A tool for addressing construct identity in literature reviews and meta analyses", MIS Q., Vol.40, (2016), pp.529-55.

[15] Castillo A, Castellanos A \& Tremblay MC, "Improving case management via statistical text mining in a foster care organization", Desrist, LNCS, vol. 8463, (2014), pp.312-320.

[16] Luther S, Berndt D, Finch D, Richardson M, Hickling E \& Hickam D, "Using statistical text mining to supplement the development of an ontology", J. Biomed. Inform., Vol.44, (2011), pp.S86-S93.

[17] Jepperson RL, "Institutions, institutional effects, and institutionalism", New Institutionalism Organ. Anal., Vol.6, (1991), $143-163$.

[18] Giddens A, "Central Problems in Social Theory: Action, Structure, and Contradiction in Social Analysis", University of California Press, Berkeley, (1979)

[19] Sewell Jr. WH, "A theory of structure: Duality, agency, and transformation”, Am. J. Soc., Vol.98, (1992), pp.1-29.

[20] Hughes EC, "The ecological aspect of institutions", Am. Sociol. Rev., Vol.1, (1936), pp.180-189.

[21] Barley SR \& Tolbert PS, "Institutionalization and structuration: Studying the links between action and institution", Organ. Stud., Vol.18, (1997), pp.93-117.

[22] G Ainabekova, Z Bayanbayeva, B Joldasbekova, A Zhaksylykov (2018). The author in esthetic activity and the functional text (on the basis of V. Mikhaylov's narrative ("The chronicle of the great jute"). Opción, Año 33. 63-80.

[23] D, Ibrayeva, Z Salkhanova, B Joldasbekova, Zh Bayanbayeva (2018). The specifics of the art autobiography genre. Opción, Año 33. 126-151. 